\title{
Effect of Low Tube Voltage on Image Quality, Radiation Dose, and Low-Contrast Detectability at Abdominal Multidetector CT: Phantom Study
}

\author{
Kun Tang, ${ }^{1}$ Ling Wang, ${ }^{2}$ Rui Li, ${ }^{1}$ Jie Lin, ${ }^{1}$ Xiangwu Zheng, ${ }^{1}$ and Guoquan Cao ${ }^{1}$ \\ ${ }^{1}$ Department of Radiology, The First Affiliated Hospital of Wenzhou Medical College, Zhejiang, \\ Wenzhou 325000, China \\ ${ }^{2}$ Department of Nuclear Medicine, Wenzhou Medical College, Zhejiang, Wenzhou 325000, China
}

Correspondence should be addressed to Xiangwu Zheng, zxwu1111@sina.com

Received 5 January 2012; Revised 19 February 2012; Accepted 24 February 2012

Academic Editor: Lie-Hang Shen

Copyright () 2012 Kun Tang et al. This is an open access article distributed under the Creative Commons Attribution License, which permits unrestricted use, distribution, and reproduction in any medium, provided the original work is properly cited.

\begin{abstract}
Purpose. To investigate the effect of low tube voltage $(80 \mathrm{kV})$ on image quality, radiation dose, and low-contrast detectability (LCD) at abdominal computed tomography (CT). Materials and Methods. A phantom containing low-contrast objects was scanned with a CT scanner at 80 and $120 \mathrm{kV}$, with tube current-time product settings at 150-650 mAs. The differences between image noise, contrast-to-noise ratio (CNR), and scores of LCD obtained with $80 \mathrm{kV}$ at $150-650 \mathrm{mAs}$ and those obtained with $120 \mathrm{kV}$ at $300 \mathrm{mAs}$ were compared respectively. Results. The image noise substantially increased with low tube voltage. However, with identical dose, use of $80 \mathrm{kV}$ resulted in higher CNR compared with CNR at $120 \mathrm{kV}$. There were no statistically significant difference in CNR and scores of LCD between $120 \mathrm{kV}$ at $300 \mathrm{mAs}$ and $80 \mathrm{kV}$ at $550-650 \mathrm{mAs}(P>0.05)$. The relative dose delivered at $80 \mathrm{kV}$ ranged from $58 \%$ at $550 \mathrm{mAs}$ to $68 \%$ at $650 \mathrm{mAs}$. Conclusion. With a reduction of the tube voltage from $120 \mathrm{kV}$ to $80 \mathrm{kV}$ at abdominal CT, the radiation dose can be reduced by $32 \%$ to $42 \%$ without degradation of CNR and LCD.
\end{abstract}

\section{Introduction}

There has been a remarkable increase in use of multidetector computed tomography (MDCT) since its introduction. MDCT has greater diagnostic capability and enables extended clinical applications, but it also has the potential to lead to an increase in radiation dose owing to the routine use of thinner sections, the extended volume of acquisition, and multiple-phase acquisitions. According to the literature, currently, CT represents about $7 \%$ of all radiologic examinations in the world but contributes more than $40 \%$ of the collective effective dose [1]. The theoretic risk to patients for radiationindeed cancer from CT examination is not negligible [2-4].

In particular, the radiation dose from hepatic CT examinations has notably increased because multiple-phase dynamic-enhanced CT scan was routinely performed in patients who are suspected of having hepatic tumors. The estimated risk of cancer death for those undergoing CT is
12.5/10,000 population for each pass of the CT scan through the abdomen [5]. Therefore, concerns regarding a reduction in radiation dose have been recently raised during abdominal CT acquisitions.

Although decreasing tube current is the most means of reducing CT radiation dose [6-9], this alteration also reduces the contrast-to-noise ratio (CNR), which may affect the diagnostic outcome of the examination. This is especially true in abdominal studies, where low-contrast areas are severely affected by the CNR [10]. Some studies [11-15] suggest that scanning with low tube voltage is possible to reduce dose without markedly affecting image quality; however, there are few reports on the effect of low tube voltage on abdominal image quality and low-contrast detectability (LCD). Thus, the purpose of this study was to investigate the effect of low tube voltage with $80 \mathrm{kV}$ on image noise (SD, standard deviation of CT number), CNR, radiation dose, and LCD at abdominal MDCT. 


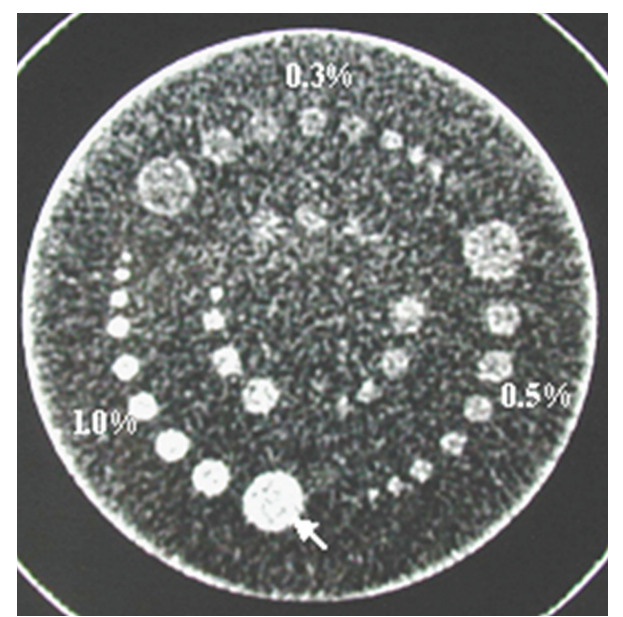

Figure 1: An axial CT image of the low-contrast module. It contains three groups of cylinders with various diameters from 2.0 to $15.0 \mathrm{~mm}$. The nominal contrast levels of these groups are $0.3 \%$, $0.5 \%$, and $1.0 \%$. In our study, only the $15.0 \mathrm{~mm}$ diameter object with a contrast difference of $1.0 \%$ (white arrow) was chosen to be analyzed.

\section{Materials and Methods}

The institutional review board approved this study, and informed consent was obtained from all participating radiologists.

2.1. Description of Phantom. We used a phantom (Catphan 500; Phantom Laboratory, Cambridge, NY) with an additional annulus provided by the manufacturer to simulate the $\mathrm{X}$-ray absorption of a standard abdomen (giving a total test object diameter of $30 \mathrm{~cm}$ ). The phantom contains a CTP515 module, which consists of a $40 \mathrm{~mm}$ thick and $200 \mathrm{~mm}$ diameter slice of tissue equivalent background material containing a series of cylinders of various diameters to measure lowcontrast performance. The cylinders varied in diameters from 2.0 to $15.0 \mathrm{~mm}$ and deviated from nominal contrast levels by $0.3 \%, 0.5 \%$, and $1.0 \%$ (Figure 1 ). In our study, in order to avoid a partial volume effect, only the $15.0 \mathrm{~mm}$ diameter object with a contrast difference of $1.0 \%$ (having an attenuation difference with the background of $10 \mathrm{HU}$ ) was chosen to be analyzed. The phantom was always positioned at the isocenter of the gantry.

2.2. CT Scanning. The phantom was scanned three times for each protocol with a 16-section MDCT scanner (LightSpeed; GE Medical Systems). The scanning parameters were configuration of 16 (detectors) $\times 1.5 \mathrm{~mm}$ (detector collimation), rotation time of 0.75 second, section thickness of $5.0 \mathrm{~mm}$, section interval of $5.0 \mathrm{~mm}$, pitch of 0.659 , scan field of view of $50 \mathrm{~cm}$, reconstruction algorithm (kernel) B30f (mediumsharp), and pixel matrix size of $512 \times 512$. Scanning was performed at the standard tube voltage of $120 \mathrm{kV}$ and at the low tube voltage of $80 \mathrm{kV}$, with corresponding tube currenttime product settings at $150,200,250,300,350,400,450$, $500,550,600$, and $650 \mathrm{mAs}$, respectively.
2.3. Measurement of Radiation Dose. We used the CT dose index volume (CTDIvol) based on the manufacturer's data for estimation of radiation dose. The corresponding CTDIvol of each acquisition conditions indicated on the monitor screen was recorded. The CTDIvol obtained at standard tube voltage protocol was compared with that obtained at low tube voltage protocol.

2.4. Measurement of CNR. For each scanning technique, we measured the CT number of the low-contrast object in $15 \mathrm{~mm}$ diameter and the background of the module. The region of interest used to perform the measurements was kept at $100 \mathrm{~mm}^{2}$. CNRs were calculated as follows: $\mathrm{CNR}=$ (ROIm - ROIb)/SDb, where ROIm and ROIb are the CT numbers of the low-contrast object in a $15 \mathrm{~mm}$ diameter region of interest and of the background region of interest, respectively, and $\mathrm{SDb}$ is the standard deviation of the attenuation values of the background [16]. A CNR was calculated on the three images of each set of acquisition parameters. The measurement was repeated three times on each image, giving nine measurements for each acquisition condition. From these nine measurements, a mean CNR was calculated for each set of acquisition conditions [17].

2.5. Assessment of LCD. For the subjective assessments of LCD, we evaluated the images obtained at $120 \mathrm{kV}$ and $300 \mathrm{mAs}$ and the images obtained at $80 \mathrm{kV}$ and $150-650 \mathrm{mAs}$. Two experienced observers who were blind to each set of scanning parameters were asked to review independently the images. The visualization of each object was graded on a 3-point scoring scale by each observer: a score of 3.0 was obtained when the object was clearly visible and appeared as a perfect circle, a score of 2.0 was obtained when the object was not clearly visible, and a score of 1.0 was obtained when the object could not be detected. A total of 36 images (twelve sets of three images each) were respectively assessed by each observer. The final score of LCD of each acquisition sets was calculated by averaging the results of the two observers. The time for reading the images was not limited, and each observer could freely adjust the window levels and window widths on the monitor screen.

2.6. Statistical Analysis. We used a two-tailed Student's $t$ test to evaluate differences in SD, CNR, and CTDIvol between scanning performed with $80 \mathrm{kV}$ and scanning performed with $120 \mathrm{kV}$. Both the relationship between SD and tube current-time product settings and the relationship between CNR and CTDIvol were investigated using the linear regression analysis and Pearson correlation coefficient $(r)$. For subjective assessment, the Mann-Whitney $U$ test was used to analyze differences in subjective scores between standard setting $(120 \mathrm{kV}, 300 \mathrm{mAs})$ and low tube voltage settings $(80 \mathrm{kV}$, 150-650 mAs). Interobserver variation was assessed using Cohen kappa statistics. Kappa values less than 0.20 indicated poor agreement; $0.21-0.40$, fair agreement; $0.41-$ 0.60 , moderate agreement; $0.61-0.80$, good agreement; $0.81-$ 1.00 , excellent agreement. All statistical analyses were performed with a commercially available software package 
TABLE 1: The CTDIvol values obtained at each set of acquisition conditions.

\begin{tabular}{lcc}
\hline \multirow{2}{*}{ Tube current-time product (mAs) } & \multicolumn{2}{c}{ CTDIvol (mGy) } \\
& $120 \mathrm{kVp}$ & $80 \mathrm{kVp}$ \\
\hline 150 & 10.19 & 3.24 \\
200 & 13.59 & 4.33 \\
250 & 16.99 & 5.41 \\
300 & 20.39 & 6.49 \\
350 & 23.55 & 7.49 \\
400 & 26.91 & 8.57 \\
450 & 30.28 & 9.64 \\
500 & 33.64 & 10.71 \\
550 & 37.00 & 11.78 \\
600 & 40.37 & 12.85 \\
650 & 43.73 & 13.92 \\
\hline
\end{tabular}

(SPSS, version 15.0), and a $P$ value of less than 0.05 was considered to be statistically significant.

\section{Results}

3.1. Radiation Dose. The CTDIvol obtained from each set of acquisition conditions is shown in Table 1. At equal tube current-time product settings, the CTDIvol obtained at $80 \mathrm{kV}$ was approximately $32 \%$ of that at $120 \mathrm{kV}$. Compared with the CTDIvol obtained at $120 \mathrm{kV}$ and $300 \mathrm{mAs}$, the relative CTDIvols obtained at $80 \mathrm{kV}$ were $16 \%$ at $150 \mathrm{mAs}$, $21 \%$ at $200 \mathrm{mAs}, 27 \%$ at $250 \mathrm{mAs}, 32 \%$ at $300 \mathrm{mAs}, 37 \%$ at $350 \mathrm{mAs}, 42 \%$ at $400 \mathrm{mAs}, 47 \%$ at $450 \mathrm{mAs}, 53 \%$ at $500 \mathrm{mAs}$, $58 \%$ at $550 \mathrm{mAs}, 63 \%$ at $600 \mathrm{mAs}$, and $68 \%$ at $650 \mathrm{mAs}$.

3.2. Image Quality Results. The results of CT numbers, image noise, and CNR at each scanning technique are listed in Table 2. As expected, the image noise was inversely correlative to tube current. At identical tube current, the lowest and the highest noise were seen at $120 \mathrm{kV}$ and $80 \mathrm{kV}$, respectively (Figure 2). Compared with the noise obtained with $120 \mathrm{kV}$ at $300 \mathrm{mAs}$, the noise obtained with $80 \mathrm{kV}$ at $150-650 \mathrm{mAs}$ was significantly higher $(P<0.001)$ (Table 2$)$. There was a direct correlation between the CNR and the CTDIvol, with Pearson correlation coefficient $r=0.95(P<0.001)$ at $80 \mathrm{kV}$ and $r=0.96(P<0.001)$ at $120 \mathrm{kV}$. At identical CTDIvol, use of $80 \mathrm{kV}$ tube voltage resulted in higher CNR compared with CNR at $120 \mathrm{kV}$. At identical CNR, the CTDIvol at $80 \mathrm{kV}$ was substantially lower than that at $120 \mathrm{kV}$ (Figure 3). By using a two-tailed Student's $t$ test, the CNRs obtained at $80 \mathrm{kV}$ and $150-500 \mathrm{mAs}$ were significantly lower than that at $120 \mathrm{kV}$ and $300 \mathrm{mAs}(P<0.05)$ (Table 2). However, there was no statistically significant difference between the CNR obtained with $120 \mathrm{kV}$ at $300 \mathrm{mAs}$ and the CNR obtained with $80 \mathrm{kV}$ at $550 \mathrm{mAs}, 600 \mathrm{mAs}$, and $650 \mathrm{mAs}(P>0.05)$ (Table 2).

3.3. LCD Results. The subjective scores of LCD assigned by two observers are shown in Table 3 . The mean score of the three images assigned at $120 \mathrm{kV}$ and $300 \mathrm{mAs}$ was $2.83 \pm$ 0.41 . At $80 \mathrm{kV}$, the mean score $(1.00 \pm 0.00$ at $150-250 \mathrm{mAs}$, $1.83 \pm 0.41$ at $300 \mathrm{mAs}, 2.00 \pm 0.00$ at $350 \mathrm{mAs}, 2.33 \pm 0.52$

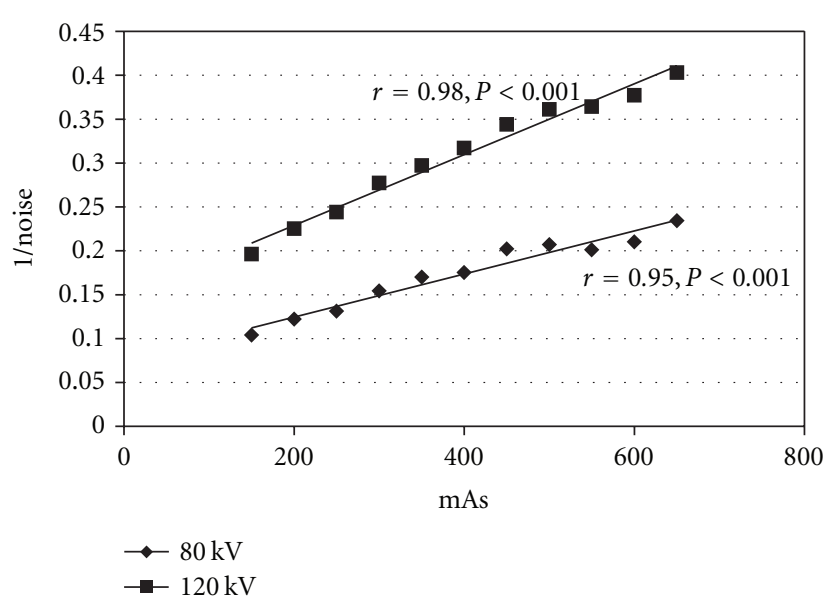

FIGURE 2: Graph shows inversely correlative between tube current (mAs) and image noise. At identical tube current, image noise obtained at $80 \mathrm{kV}$ is higher than that at $120 \mathrm{kV}$.

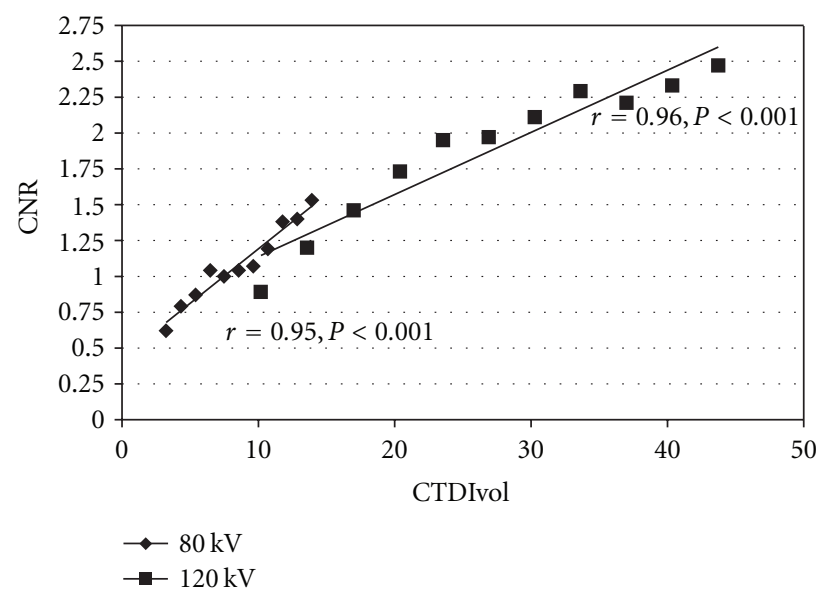

FIGURE 3: Graph shows relationship between CTDIvol and CNR. The Pearson correlation coefficient $(r)$ and the corresponding $P$ values were $r=0.95$ and $P<0.001$ at $80 \mathrm{kV}$ and $r=0.96$ and $P<0.001$ at $120 \mathrm{kV}$.

at $400-500 \mathrm{mAs}$ ) was significantly lower than that at $120 \mathrm{kV}$ and $300 \mathrm{mAs}(P=0.001$ at $150-350 \mathrm{mAs} ; P=0.019$ at 400 $500 \mathrm{mAs}$ ) (Table 3 ). However, there was no statistically significant difference between the mean score at $120 \mathrm{kV}$ and $300 \mathrm{mAs}$ and the mean score assigned at $80 \mathrm{kV}$ and the other tube current settings that we investigated $(P=0.138$ at $550 \mathrm{mAs} ; P=0.317$ at $600 \mathrm{mAs} ; P=1.0$ at $650 \mathrm{mAs}$ ) (Table 3). Using Cohen kappa statistics, the interobserver agreement in regard to subjective assessment of LCD was $\operatorname{good}(\kappa=0.67)$.

\section{Discussion}

Improvement in MDCT technology now allows CT examinations to be easily and fast performed, leading to a possible increase of the radiation dose to patients. In particular, the radiation exposure and risk of cancer death from hepatic CT examinations have notably increased because multiple-phase 
TABLE 2: The CT numbers, image noise, and CNR obtained at each set of acquisition conditions.

\begin{tabular}{lccccccccc}
\hline \multirow{2}{*}{ Tube current-time product (mAs) } & \multicolumn{2}{c}{ CT number } & \multirow{2}{*}{$P$ value } & \multicolumn{2}{c}{ Image noise } & \multicolumn{2}{c}{$P$ value* } & \multicolumn{2}{c}{ CNR } \\
& $120 \mathrm{kV}$ & $80 \mathrm{kV}$ & & $120 \mathrm{kV}$ & $80 \mathrm{kV}$ & & \multicolumn{2}{c}{$P$ value* } \\
\hline 150 & 56.12 & 27.56 & $<0.001$ & 5.10 & 9.60 & $<0.001$ & 0.89 & 0.62 & $<0.001$ \\
200 & 56.34 & 27.73 & $<0.001$ & 4.45 & 8.20 & $<0.001$ & 1.20 & 0.79 & $<0.001$ \\
250 & 56.21 & 28.17 & $<0.001$ & 4.09 & 7.61 & $<0.001$ & 1.46 & 0.87 & 0.006 \\
300 & 56.76 & 28.35 & $<0.001$ & 3.61 & 6.50 & $<0.001$ & 1.73 & 1.04 & 0.029 \\
350 & 56.93 & 28.21 & $<0.001$ & 3.37 & 5.88 & $<0.001$ & 1.95 & 1.00 & 0.025 \\
400 & 56.77 & 28.41 & $<0.001$ & 3.15 & 5.70 & $<0.001$ & 1.97 & 1.04 & 0.028 \\
450 & 56.69 & 28.43 & $<0.001$ & 2.91 & 4.96 & $<0.001$ & 2.11 & 1.07 & 0.031 \\
500 & 57.00 & 28.36 & $<0.001$ & 2.77 & 4.82 & $<0.001$ & 2.29 & 1.19 & 0.042 \\
550 & 56.78 & 28.68 & $<0.001$ & 2.75 & 4.97 & $<0.001$ & 2.21 & 1.38 & 0.224 \\
600 & 57.14 & 28.78 & $<0.001$ & 2.65 & 4.76 & $<0.001$ & 2.33 & 1.40 & 0.272 \\
650 & 57.14 & 28.86 & $<0.001$ & 2.48 & 4.27 & $<0.001$ & 2.47 & 1.53 & 0.501 \\
\hline
\end{tabular}

* The $P$ values are those obtained with $80 \mathrm{kV}$ at the $150-650 \mathrm{mAs}$ settings compared with the values obtained with $120 \mathrm{kV}$ and $300 \mathrm{mAs}$.

TABLE 3: The subjective scores of LCD.

\begin{tabular}{|c|c|c|c|c|}
\hline \multirow{2}{*}{ Tube voltage/tube current-time product } & \multicolumn{2}{|c|}{ Subjective score of LCD } & \multirow{2}{*}{ Mean } & \multirow{2}{*}{$P$ value* } \\
\hline & Observer A & Observer B & & \\
\hline $80 \mathrm{kV} / 150 \mathrm{mAs}$ & $3.0(1.0,1.0,1.0)$ & $3.0(1.0,1.0,1.0)$ & $1.00 \pm 0.00$ & 0.001 \\
\hline $80 \mathrm{kV} / 200 \mathrm{mAs}$ & $3.0(1.0,1.0,1.0)$ & $3.0(1.0,1.0,1.0)$ & $1.00 \pm 0.00$ & 0.001 \\
\hline $80 \mathrm{kV} / 250 \mathrm{mAs}$ & $3.0(1.0,1.0,1.0)$ & $3.0(1.0,1.0,1.0)$ & $1.00 \pm 0.00$ & 0.001 \\
\hline $80 \mathrm{kV} / 300 \mathrm{mAs}$ & $5.0(1.0,2.0,2.0)$ & $6.0(2.0,2.0,2.0)$ & $1.83 \pm 0.41$ & 0.001 \\
\hline $80 \mathrm{kV} / 350 \mathrm{mAs}$ & $6.0(2.0,2.0,2.0)$ & $6.0(2.0,2.0,2.0)$ & $2.00 \pm 0.00$ & 0.001 \\
\hline $80 \mathrm{kV} / 400 \mathrm{mAs}$ & $7.0(2.0,3.0,2.0)$ & $7.0(2.0,3.0,2.0)$ & $2.33 \pm 0.52$ & 0.019 \\
\hline $80 \mathrm{kV} / 450 \mathrm{mAs}$ & $7.0(3.0,2.0,2.0)$ & $7.0(2.0,2.0,3.0)$ & $2.33 \pm 0.52$ & 0.019 \\
\hline $80 \mathrm{kV} / 500 \mathrm{mAs}$ & $8.0(2.0,3.0,3.0)$ & $6.0(2.0,2.0,2.0)$ & $2.33 \pm 0.52$ & 0.019 \\
\hline $80 \mathrm{kV} / 550 \mathrm{mAs}$ & $8.0(3.0,3.0,2.0)$ & $8.0(3.0,2.0,3.0)$ & $2.67 \pm 0.52$ & 0.138 \\
\hline $80 \mathrm{kV} / 600 \mathrm{mAs}$ & $9.0(3.0,3.0,3.0)$ & $8.0(2.0,3.0,3.0)$ & $2.83 \pm 0.41$ & 0.317 \\
\hline $80 \mathrm{kV} / 650 \mathrm{mAs}$ & $9.0(3.0,3.0,3.0)$ & $9.0(3.0,3.0,3.0)$ & $3.00 \pm 0.00$ & 1.000 \\
\hline $120 \mathrm{kV} / 300 \mathrm{mAs}$ & $9.0(3.0,3.0,3.0)$ & $9.0(3.0,3.0,3.0)$ & $3.00 \pm 0.00$ & - \\
\hline
\end{tabular}

* The $P$ values are those obtained with $80 \mathrm{kV}$ at the $150-650 \mathrm{mAs}$ settings compared with the values obtained with $120 \mathrm{kV}$ and $300 \mathrm{mAs}$. There was good agreement between observer $A$ and observer $B$ in regard to subjective assessment of LCD $(\kappa=0.67)$.

dynamic-enhanced CT scan is routinely performed. Managing patient dose is therefore a major concern in abdominal MDCT examinations.

In our study, we used the CT dose index volume (CTDIvol) based on the manufacturer's data for estimation of radiation dose. CTDI, expressed in terms of air kerma in milligray, was obtained at the periphery (CTDIp) and at the centre (CTDIc) of a special $100 \mathrm{~mm}$ long pencil-shaped ionisation chamber. The weighted CTDIw is obtained as the sum of one-third of CTDIc and two-thirds of CTDIp. The CTDIvol, which is CTDIw divided by the pitch, represents the average volume dose (air kerma) within a specified CT dosimetry phantom [18]. CTDIvol is a good measure of CT radiation dose for applications where the patient table is incremented during the scan. Adoption of CTDIvol as the intensity of the radiation dose would facilitate accurate comparisons of radiation doses used for different tube voltages. For instance, in our study, it is easy for us to compare the difference of radiation dose between $80 \mathrm{kV}$ and $120 \mathrm{kV}$ tube voltage. Results of our study showed that it was possible to reduce radiation exposure substantially by decreasing the tube voltage from $120 \mathrm{kV}$ to $80 \mathrm{kV}$. However, it has limitations. Because the CTDIvol is an averaged dose to a homogeneous cylindrical phantom, the measurements are only an approximation of patient dose. Another limitation is that CTDIvol phantom does not provide a sufficiently long scatter path relative to the typical length of a human; hence, patient dose may be underestimated with CTDIvol [19]. Therefore, the results of radiation dose based on the CTDIvol in our study could not be accurate represented patient dose. Furthermore, the difference of the radiation dose between the central and peripheral cavities of the phantom also could not be discerned by using the CTDIvol as estimation of radiation dose.

In present study, our findings showed that there was a direct correlation between the CNR and the CTDIvol, which was consistent with previous studies [16, 20]. Although the mean CNR was decreased when CT acquisition was performed at a tube voltage of $80 \mathrm{kV}$ and an identical tube current setting, CNR improved substantially when identical CTDIvol was used. Compared with CNR obtained at $120 \mathrm{kV}$ 
and $300 \mathrm{mAs}$, there was no statistically significant difference at $80 \mathrm{kV}$ and $550 \mathrm{mAs}, 600 \mathrm{mAs}$, and $650 \mathrm{mAs}(P>0.05)$. This suggest that image quality including CNR acquired at $80 \mathrm{kV}$ with tube current higher than $550 \mathrm{mAs}$ is equivalent to that acquired at $120 \mathrm{kV}$ and $300 \mathrm{mAs}$. Furthermore, the relative radiation dose obtained at $80 \mathrm{kV}$ and $550 \mathrm{mAs}, 600 \mathrm{mAs}$, and $650 \mathrm{mAs}$ was $58 \%, 63 \%$, and $68 \%$ of that at $120 \mathrm{kVp}$ and $300 \mathrm{mAs}$, respectively. Therefore, we postulate that scanning with a low tube voltage as low as $80 \mathrm{kV}$ is feasible in abdominal CT examination without loss of diagnostic accuracy when the tube current is higher than $550 \mathrm{mAs}$ allowing reduction in the radiation dose by $32 \%$ to $42 \%$.

LCD is one of the most important factors in abdominal $\mathrm{CT}$, especially when looking for small lesions in abdominal organs such as liver, spleen, pancreas, or kidneys. This LCD is not only relevant for unenhanced series but also contrast enhanced series, as contrast between normal and abnormal tissue may be only slightly increased by iodine [21]. Awai et al. [22] reported that in enhanced hepatic CT, tumorto-liver contrast was 5-40 HU. In present study, the object with a contrast difference of $1.0 \%$ (having an attenuation difference with the background of $10 \mathrm{HU}$ ) was chosen to be analyzed. When the two experienced observers subjectively assessed the visibilities of low-contrast images, the subjective scores of LCD assigned at $80 \mathrm{kV}$ and 550, 600, and $650 \mathrm{mAs}$ did not differ significantly from those assigned at $120 \mathrm{kV}$ and $300 \mathrm{mAs}$. Furthermore, the mean score of LCD obtained at $80 \mathrm{kV}$ and $650 \mathrm{mAs}$ was slightly higher than that at $120 \mathrm{kV}$ and $300 \mathrm{mAs}$. These results suggested that a reduction from $120 \mathrm{kV}$ to $80 \mathrm{kV}$ also could result in up to $42 \%$ dose reduction without compromising LCD. Funama et al. [16] showed that a $35 \%$ reduction in the radiation dose could be achieved when scanning was performed at $90 \mathrm{kV}$ rather than at $120 \mathrm{kV}$ without degradation of LCD. Our findings agree with Funama and suggest that lower tube voltage can be used in abdominal CT thereby achieving dose reduction while maintaining acceptable image quality. In this study, we found no statistically significant difference in both CNR and LCD at $120 \mathrm{kV}$ and $300 \mathrm{mAs}$ compared with those at $80 \mathrm{kV}$ and 550-650 mAs. This is probably because CNR and LCD are parallel to each other, which is consistent with Verdun's result [17]. He found that there was a significant correlation between the mean CNR measurements and the subjective scores of LCD $(r=0.95, P<0.05)$.

The main drawback of the low tube voltage technique is the increase in image noise caused by the reduced photon flux. In our study, we found that the noise values obtained with $80 \mathrm{kV}$ at the $150-650 \mathrm{mAs}$ settings were significant higher than that obtained with $120 \mathrm{kV}$ and $300 \mathrm{mAs}(P<$ $0.001)$. As previously reported $[10,20]$, we found that there was an inversely correlative relationship between the image noise and the tube current. In another word, the increased noise will be obtained when the strategy of lower tube current or lower tube voltage is implemented. Image noise, however, has a greater effect on the quality of abdominal images because the abdominal region is inherently of lower contrast. Therefore, for CT scanning with low tube voltage, higher tube current settings are required to compensate for the lower number of photons. In addition, some new techniques should be developed to reduce image noise. Several articles in the last years have been reported that noise reduction filters [23-25] as well as reconstruction methods, such as adaptive statistical iterative reconstruction [26-28] could effectively help to reduce the noise on CT images with radiation dose reduction without compromise of image quality.

4.1. Study Limitations. We acknowledge that this study contains certain limitations. First, this CT scanning with low tube voltage at $80 \mathrm{kV}$ was only performed in a phantom study, and the phantom did not consider variability of body composition, therefore, whether this result is suitable to clinical using needs to be further confirmed. However, Marin et al. [14] showed that a technique with low tube voltage at $80 \mathrm{kV}$ could be applied to improve the conspicuity of malignant hypervascular liver tumors while significantly reducing patient radiation dose. Secondly, our investigation did not take into account differences in body sizes. Attenuation of the incident X-ray beam in CT depends on the size of body portion being evaluated; that is, greater exposure is required in corpulent patients to attain image quality equal to that in slimmer patients [29]. Although studies in patients were not part of this investigation, previous studies with a phantom suggest that the technique is effective for dose reduction of abdominal CT for relatively light weight patients whose body weight is less than $80 \mathrm{~kg}$ [30]. Another important aspect is that many patients present with high-attenuation implants, which can dramatically decrease image quality when low $\mathrm{kV}$ protocols are used routinely. Finally, we only used the CTDIvol provided by the manufacturer to estimate the radiation dose. Although the agreement between the values provided by the manufacturer and the measured values was good, with differences of less than $10 \%$ [31], there were some limitations as mentioned above.

\section{Conclusions}

In this CT phantom study we have shown that although image noise is increased at low tube voltage, it is possible to reduce radiation dose by up to $42 \%$ without degradation of CNR and LCD by reducing tube voltage from 120 to $80 \mathrm{kV}$ and increasing tube current to more than $550 \mathrm{mAs}$. As an effective technique of reducing CT radiation dose, scanning with low tube voltage would benefit patients with relatively light weight, especially those who may need to undergo MDCT examinations for long-term followup or high-risk screening.

\section{References}

[1] F. A. Mettler Jr., M. Bhargavan, K. Faulkner et al., "Radiologic and nuclear medicine studies in the United States and worldwide: frequency, radiation dose, and comparison with other radiation sources-1950-2007," Radiology, vol. 253, no. 2, pp. 520-531, 2009.

[2] D. J. Brenner and C. D. Elliston, "Estimated radiation on risks potentially associated with full-body CT screening," Radiology, vol. 232, no. 3, pp. 735-738, 2004. 
[3] D. J. Brenner, "Radiation risks potentially associated with lowdose CT screening of adult smokers for lung cancer," Radio$\log y$, vol. 231, no. 2, pp. 440-445, 2004.

[4] A. B. De González and S. Darby, "Risk of cancer from diagnostic X-rays: estimates for the UK and 14 other countries," Lancet, vol. 363, no. 9406, pp. 345-351, 2004.

[5] V. Tsapaki, M. Rehani, and S. Saini, "Radiation safety in abdominal computed tomography," Seminars in Ultrasound, CT and MRI, vol. 31, no. 1, pp. 29-38, 2010.

[6] S. A. Sohaib, P. D. Peppercorn, J. A. Horrocks, M. H. Keene, G. S. Kenyon, and R. H. Reznek, "The effect of decreasing mAs on image quality and patient dose in sinus CT," British Journal of Radiology, vol. 74, no. 878, pp. 157-161, 2001.

[7] L. M. Hamberg, J. T. Rhea, G. J. Hunter, and J. H. Thrall, "Multi-detector row CT: radiation dose characteristics," Radiology, vol. 226, no. 3, pp. 762-772, 2003.

[8] D. P. Frush, C. C. Slack, C. L. Hollingsworth et al., "Computer-simulated radiation dose reduction for abdominal multidetector CT of pediatric patients," American Journal of Roentgenology, vol. 179, no. 5, pp. 1107-1113, 2002.

[9] A. F. Kopp, M. Heuschmid, and C. D. Claussen, "Multidetector helical CT of the liver for tumor detection and characterization," European Radiology, vol. 12, no. 4, pp. 745-752, 2002.

[10] "Managing patient dose in computed tomography. A report of the International Commission on Radiological Protection," Annals of the ICRP, vol. 30, no. 4, pp. 7-45, 2000.

[11] J. Hausleiter, S. Martinoff, M. Hadamitzky et al., "Image quality and radiation exposure with a low tube voltage protocol for coronary CT angiography: results of the PROTECTION II Trial," Cardiovascular Imaging, vol. 3, no. 11, pp. 1113-1123, 2010.

[12] D. Marin, R. C. Nelson, H. Barnhart et al., "Detection of pancreatic tumors, image quality, and radiation dose during the pancreatic parenchymal phase: effect of a low-tube-voltage, high-tube-current CT technique-preliminary results," Radiology, vol. 256, no. 2, pp. 450-459, 2010.

[13] S. T. Schindera, R. C. Nelson, S. Mukundan et al., "Hypervascular liver tumors: low tube voltage, high tube current multidetector row CT for enhanced detection-phantom study," Radiology, vol. 246, no. 1, pp. 125-132, 2008.

[14] D. Marin, R. C. Nelson, E. Samei et al., "Hypervascular liver tumors: low tube voltage, high tube current multidetector CT during late hepatic arterial phase for detection-initial clinical experience," Radiology, vol. 251, no. 3, pp. 771-779, 2009.

[15] B. Bischoff, F. Hein, T. Meyer et al., "Impact of a reduced tube voltage on CT angiography and radiation dose. Results of the PROTECTION I Study," Cardiovascular Imaging, vol. 2, no. 8, pp. 940-946, 2009.

[16] Y. Funama, K. Awai, Y. Nakayama et al., "Radiation dose reduction without degradation of low-contrast detectability at abdominal multisection CT with a low-tube voltage technique: phantom study," Radiology, vol. 237, no. 3, pp. 905-910, 2005.

[17] F. R. Verdun, A. Denys, J. F. Valley, P. Schnyder, and R. A. Meuli, "Detection of low-contrast objects: experimental comparison of single- and multi-detector row CT with a phantom1," Radiology, vol. 223, no. 2, pp. 426-431, 2002.

[18] C. H. McCollough, M. R. Bruesewitz, M. F. McNitt-Gray et al., "The phantom portion of the American College of Radiology (ACR) Computed Tomography (CT) accreditation program: practical tips, artifact examples, and pitfalls to avoid," Medical Physics, vol. 31, no. 9, pp. 2423-2442, 2004.

[19] R. L. Dixon and K. E. Ekstrand, "A film dosimetry system for use in computed tomography," Radiology, vol. 127, no. 1, pp. 255-258, 1978.
[20] A. Waaijer, M. Prokop, B. K. Velthuis, C. J. G. Bakker, G. A. P. De Kort, and M. S. Van Leeuwen, "Circle of Willis at CT angiography: dose reduction and image quality_reducing tube voltage and increasing tube current settings," Radiology, vol. 242, no. 3, pp. 832-839, 2007.

[21] H. J. Brisse, J. Brenot, N. Pierrat et al., "The relevance of image quality indices for dose optimization in abdominal multidetector row CT in children: experimental assessment with pediatric phantoms," Physics in Medicine and Biology, vol. 54, no. 7, pp. 1871-1892, 2009.

[22] K. Awai, K. Takada, H. Onishi, and S. Hori, "Aortic and hepatic enhancement and tumor-to-liver contrast: analysis of the effect of different concentrations of contrast material at multidetector row helical CT," Radiology, vol. 224, no. 3, pp. 757$763,2002$.

[23] Y. Yanaga, K. Awai, Y. Funama et al., "Low-dose MDCT urography: feasibility study of low-tube-voltage technique and adaptive noise reduction filter," American Journal of Roentgenology, vol. 193, no. 3, pp. W220-W229, 2009.

[24] M. K. Kalra, M. M. Maher, D. V. Sahani et al., "Low-dose CT of the abdomen: evaluation of image improvement with use of noise reduction filters-pilot study," Radiology, vol. 228, no. 1, pp. 251-256, 2003.

[25] M. K. Kalra, M. M. Maher, M. A. Blake et al., "Detection and characterization of lesions on low-radiation-dose abdominal CT images postprocessed with noise reduction filters," Radiology, vol. 232, no. 3, pp. 791-797, 2004.

[26] M. Yanagawa, O. Honda, S. Yoshida et al., "Adaptive statistical iterative reconstruction technique for pulmonary CT. Image quality of the cadaveric lung on standard- and reduced-dose CT," Academic Radiology, vol. 17, no. 10, pp. 1259-1266, 2010.

[27] S. Singh, M. K. Kalra, M. D. Gilman et al., "Adaptive statistical iterative reconstruction technique for radiation dose reduction in chest CT: a pilot study," Radiology, vol. 259, no. 2, pp. 565-573, 2011.

[28] Y. Sagara, A. K. Hara, W. Pavlicek, A. C. Silva, R. G. Paden, and Q. Wu, "Abdominal CT: comparison of low-dose CT with adaptive statistical iterative reconstruction and routine-dose CT with filtered back projection in 53 patients," American Journal of Roentgenology, vol. 195, no. 3, pp. 713-719, 2010.

[29] J. R. Haaga, "Radiation dose management: weighing risk versus benefit," American Journal of Roentgenology, vol. 177, no. 2, pp. 289-291, 2001.

[30] Y. Nakayama, K. Awai, Y. Funama et al., "Abdominal CT with low tube voltage: preliminary observations about radiation dose, contrast enhancement, image quality, and noise," Radiology, vol. 237, no. 3, pp. 945-951, 2005.

[31] F. R. Verdun, N. Theumann, P. A. Poletti et al., "Impact of the introduction of 16-row MDCT on image quality and patient dose: phantom study and multi-centre survey," European Radiology, vol. 16, no. 12, pp. 2866-2874, 2006. 


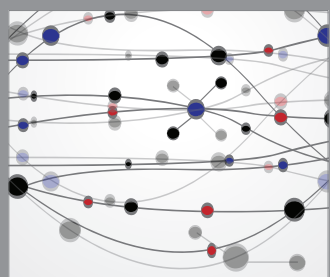

The Scientific World Journal
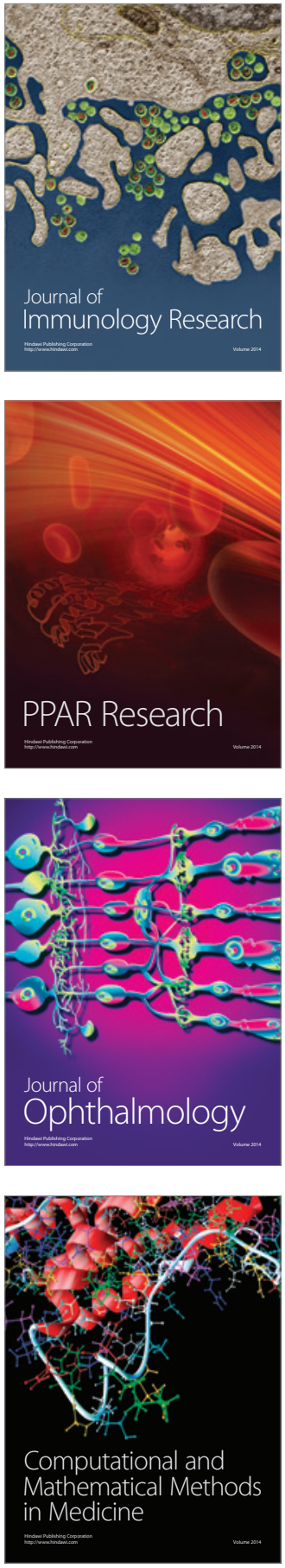

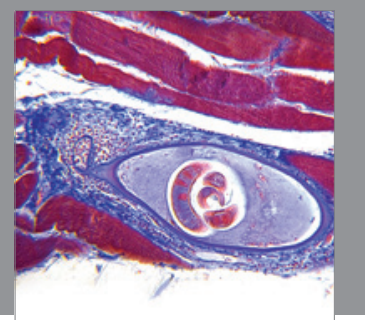

Gastroenterology

Research and Practice
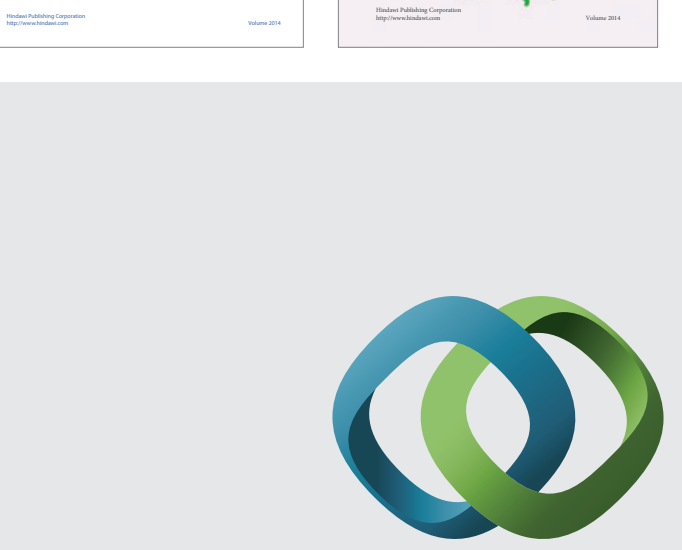

\section{Hindawi}

Submit your manuscripts at

http://www.hindawi.com
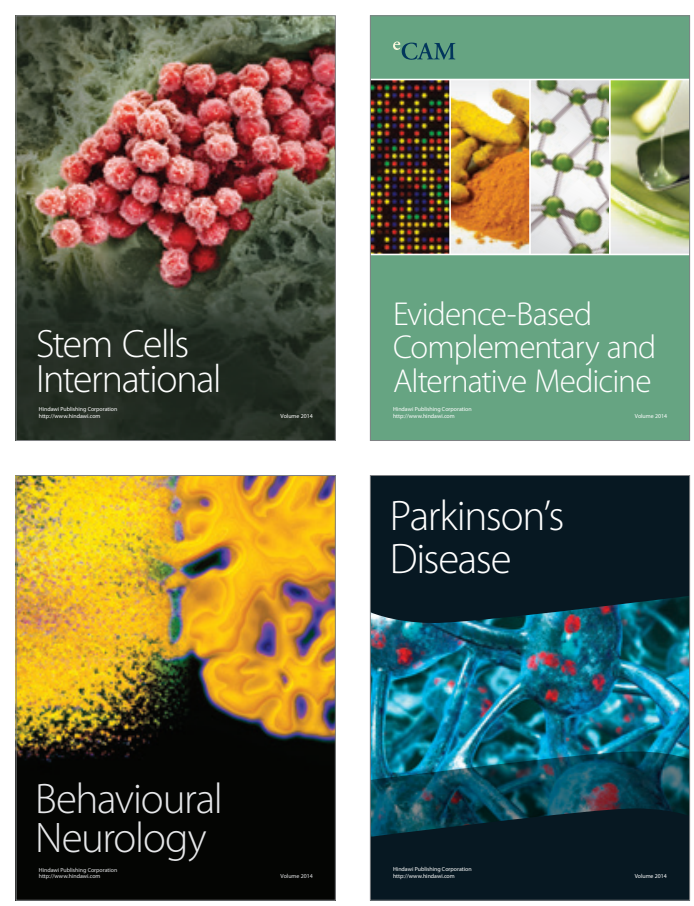

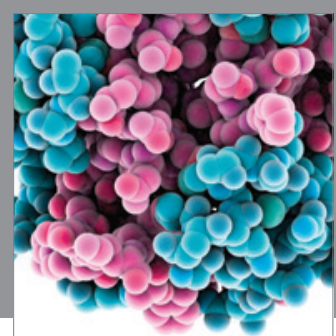

Journal of
Diabetes Research

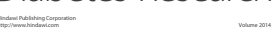

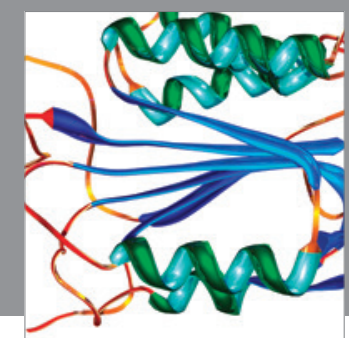

Disease Markers
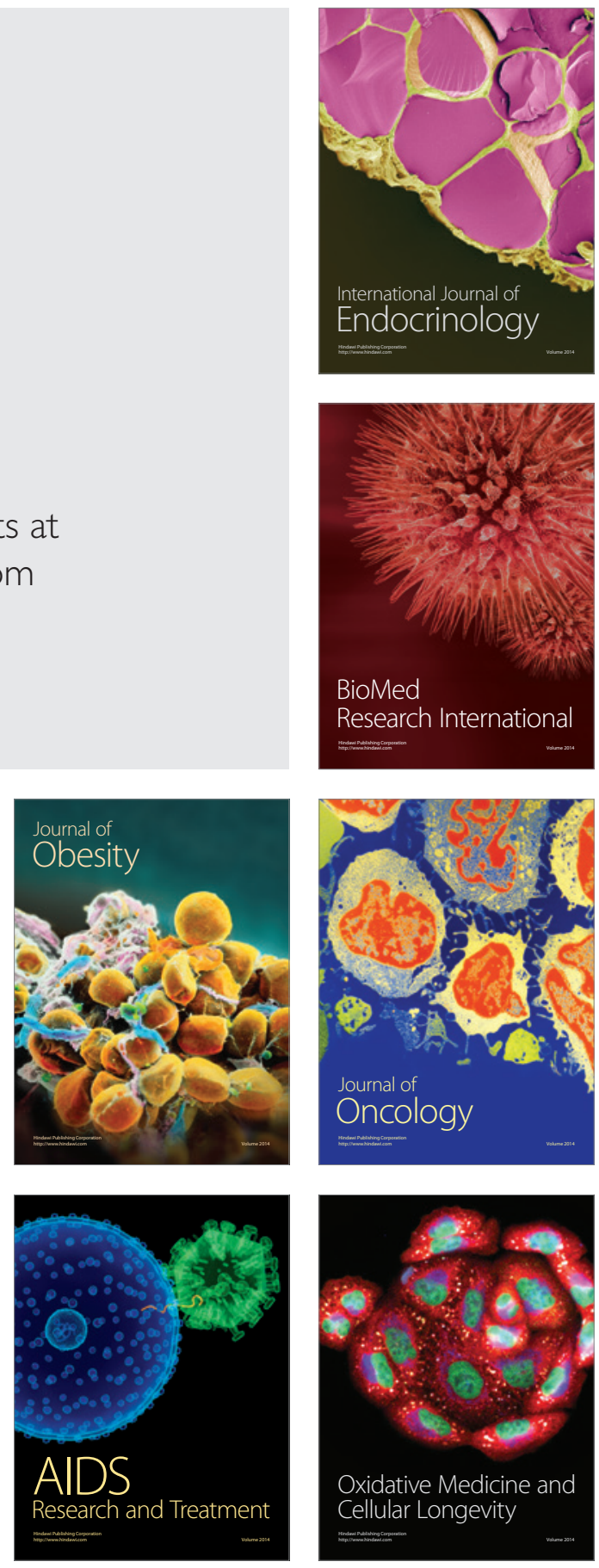